\title{
ORIENTATION IN THE PERCEPTION OF SPACE*
}

\author{
CHIZU SATO
}

Keio University

\section{Problem}

The process of orientation is involved in various kinds of behavior in men and animals. A number of observations have been reported as to the excellent ability in orientation displayed by some natives, animals, and migratory birds (Fisher, 1931). The geographical orientation of men in general has also been subjects of experimental studies. For example, Angyal (1930), Claparède (1903, 1924, 1943), Rayan (1940) and others endeavored to clarify cues being utilized by men in orientation or to discover the individual differences in apprehending spatial relationships in the geographical field. The orientation in immediate perceptive field of more limited scope has been experimentally studied by Sandström (1951). $\mathrm{He}$ is of opinion that the orientation in experiments conducted in the laboratory may have a close relationship to the orientation in geographical field of large scale.

It seems to the author that experiments concerning the orientation in men which hitherto been made are of two kinds: (1) The orientation accompanied by active or passive change of body position and (2) The orientation involving no change of body position (Sandström, 1951). In either case, the visual perception, bodily movement, kinesthesis and memory (Sato, 1960) have been discovered to play an

* This article is based on the author's Master's thesis at the Keio University. Thanks are given to Prof. T. Ogawa for his advice throughout the course of the investigation. Professors M. Yokoyama and $\mathrm{T}$. Indow kindly read the manuscript and gave helpful criticisms. important role.

In the present study, the experiments were designed in order to analyse the process of orientation under two conditions mentioned above. Exp. I dealt with the orientation of $S$ in determining the route to proceed in a room following the predetermined path in the map, whereas, Exp. II concerned with the orientation in reproducing visually presented diagrams in rotated forms.

\section{EXPERIMENT I}

\section{Method}

(1) Subjects: The $S$ s employed in Exp. I were nine university students, four males and five females, majoring in psychology.

(2) Materials: The $S$ was given one of ten maps shown in Fig. 1 and instructed to walk in a room through the indicated path in the map. The maps were made after the pattern of those used in the study of Semmes and others (1955). Each map was made of white cardboard on which the path was drawn with black lines $1.0 \mathrm{~mm}$. in width. The circle on the map indicated the starting point and triangle marked the goal. The small arrowhead at the starting point indicated the direction that the $S$ was to face when the $S$ stood at that point. Each map was square plaque, $10 \mathrm{~cm}$. on a side. The number of turns involved in the map varied from 6 to 10, and pairs such as the map No. 1 and No. 2; No. 3 and No. 4; and so forth had the same number of turns.

(3) Procedures: The general instructions given to the $S$ were as follows: "You are standing now at the starting point that is shown by the circle on the map, and facing to the direction that the arrowhead shows. You will be expected to walk along on the floor as indicated 
No. 1

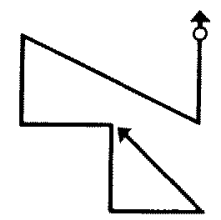

No. 3

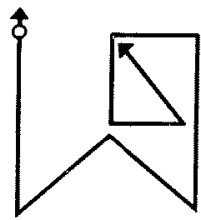

No. 5

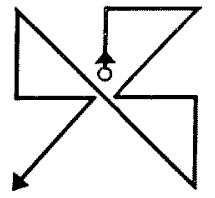

No. 7

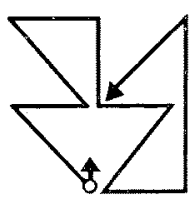

No. 9
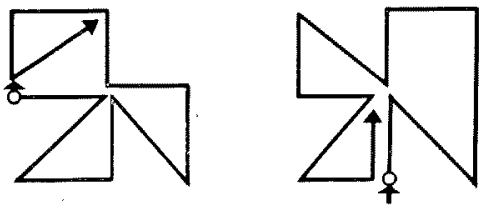

A shows the starting point and the direction $\delta$ that the $s$ was to face

4 shows the goal.

Fig. 1. The ten maps used in Exp. I.

by the path marked out on the map. Start from here and walk until you arrive at the goal, namely, the end marked by the triangle in the map. And you have to hold the map, while walking, in a constant relation with respect to your body, even though it will not always be in the proper orientation to the room. Walk directly forward-do not walk backward or sideways. When you reach a turning point, do not turn until you decide to which direction you are to go next. When you notice that you have made an incorrect turn, if it is before reaching the next comer, you may correct the turn after reporting it to the $E$. Keep in mind the proportion among lengths of the straight parts of the course and that among sizes of the angle to turn. Walk through this distance by eight steps (pointing out the line that was drawn $10 \mathrm{~cm}$. long, for example, from the starting point to the first turn of the map No. 3)."

While walking, the $S$ kept the map mounted on a board in front of him, in a constant position with respect to his body. As the $S$ was not allowed to rotate the map, it was often incorrectly oriented to actual direction in the room so that the path indicated in the map and the path through which the $S$ was to move in the room did not coincide. Hence the $S$ had to effect a translation of the spatial coordinates of the map into the corresponding actual coordinates of the room in order to reproduce the path correctly. The performances on the ten maps were carried out by all the $S$ s in each of the three experiments as follows.

Exp. A. The $S$ was instructed to walk in a normally illuminated room along the path indicated in the map.

Exp. B. The $S$ was instructed to walk in a dark room in which there was one guide lamp* on the wall $80 \mathrm{~cm}$. high from the floor. The guide lamp assumed the same shape irrespective of the position of the $S$ in the room.

Exp. C. The $S$ was instructed to walk in a completely dark room. In Exps. B and C, a small lamp which was to illuminate only the map was attached to the $S$ on the back waist, and these lights helped the $E$ to trace the $S$ 's course of walking. The area of the room in which the $S$ was to walk was about $10 \times 10 \mathrm{~m}$.

* A red miniature bulb contained in a cylinder standing vertically served as the guide lamp, which was seen through a transparent ring in the middle of the cylinder. 
in all experiments.

To prevent the $S$ from obtaining cue to orientation from memory of the position of the entrance-door, in Exps. $\mathrm{B}$ and $\mathrm{C}$, the $S$ was blindfolded when entering into the dark room and led by the $E$ to various parts of the room for about twenty sec. Then the $S$ was unblindfolded at the starting position and given the instructions as described above. After a while, the $S$ declared "start" and began to walk. When reaching the goal, the $S$ said " end" standing still with his eyes shut. Again, the $E$ led the $S$ to the next starting position and gave the $S$ the next map. The $E$ did not utter a word in order to prevent the $S$ from getting a cue to orientation from the position of the $E$.

In Exp. B, the $S$ was told that the guide lamp was in a fixed position throughout the experiment.

The $E$ sat at the middle of one side of the room and described on the paper the course of the $S$ 's movement. The time required for the $S$ to complete the whole course of one map was also recorded.

Nine $S$ s were divided into three groups of equal number. Each group participated in all the three experiments. The temporal order of the three experiments were varied from group to group. In a sitting only one experiment was conducted and ten maps were given to the $S$ in random order.

\section{Results}

(1) Comparisons among the experiments: The mean number of maps in which one or more errors were made by the $S$ in each group in each experiment is shown in Table 1. It was counted as an error when the $S$ did some wrong turn, for example, when the $S$ turned to the left at the corner where he had to turn to the right (a R-L error), or when the $S$ turned deviating by more than 30 degrees from the correct angle (an angle error). In the column headed "error" is shown the number of the maps in which an error, either the right-left error or the angle error, was observed. The figure in " error" is not necessarily equal to the sum of the figures in the remaining two columns because both kinds of errors may occur in the same map.

The number of "errors" increased, as is clear from Table 1, in the following order: Exp. A (the normally illuminated room), Exp. B (the dark room with a guide lamp), and Exp. C (the dark room). As to "error" as well as "angle error", the analysis of variance was carried out with the result that differences among the experiments were significant at $1 \%$ level. But differences among the three groups, that is, differences due to the temporal order of the experimentation were not significant.

The number of angle errors increased in the following order; Exp. A, B, and C. And differences among the experiments were greater with respect to the angle error than to the right-left error.

Table 1

The mean number of maps in which one or more errors were made by the $S$, "error" refers to both kinds treated separately in the remaining two columns.

\begin{tabular}{|c|c|c|c|c|c|c|c|c|c|c|c|c|}
\hline & \multicolumn{3}{|c|}{$\begin{array}{c}\text { Exp. A } \\
\text { illuminated room }\end{array}$} & \multicolumn{3}{|c|}{$\begin{array}{l}\text { Exp. B } \\
\text { dark room with a } \\
\text { guide lamp }\end{array}$} & \multicolumn{3}{|c|}{$\begin{array}{c}\text { Exp. C } \\
\text { dark room }\end{array}$} & \multicolumn{3}{|c|}{ Total } \\
\hline & error & R-L & angle & error & R-L & angle & error & R-L & angle & error & R-L & angle \\
\hline Gr. I & 5.3 & 4.7 & 2.7 & 7.7 & 4.0 & 6.3 & 8.0 & 5.0 & 7.3 & 21.0 & 13.7 & 16.3 \\
\hline Gr. II & 2.7 & 2.3 & 0.7 & 4.0 & 1.3 & 3.0 & 8.3 & 2.3 & 8.0 & 15.0 & 5.9 & 11.7 \\
\hline Gr. III & 2.7 & 2.7 & 0.7 & 7.0 & 4.0 & 4.0 & 7.7 & 3.7 & 6.0 & 17.4 & 10.4 & 10.7 \\
\hline Total & 10.7 & 9.7 & 4.1 & 18.7 & 9.3 & 13.3 & 24.0 & 11.0 & 21.3 & 53.4 & 30.0 & 38.7 \\
\hline
\end{tabular}


Table 2

The mean time and standard deviation of the nine $S$ s required for the ten maps in min.

\begin{tabular}{c|r|r|r}
\hline & Exp. A & Exp. B & Exp. G \\
\hline mean & 11.06 & 12.86 & 15.43 \\
S.D. & 2.81 & 3.81 & 4.56
\end{tabular}

Table 3

Number of $S_{s}$ who made one or more errors in each map in the three experiments, and the mean time required in sec.

\begin{tabular}{c|c|c}
\hline Map No. & Error & Mean time \\
\hline 1 & 16 & 72.8 \\
2 & 12 & 65.6 \\
3 & 9 & 68.7 \\
4 & 17 & 75.5 \\
5 & 21 & 86.3 \\
6 & 16 & 53.6 \\
7 & 14 & 93.9 \\
8 & 23 & 96.3 \\
9 & 19 & 93.3 \\
10 & 13 & 96.4 \\
\hline
\end{tabular}

From these findings, it is apparent that the control of angles to turn was most difficult for the $S$ in the dark room, and in Exp. B the guide lamp was somewhat useful in helping the $S$ to turn correct angles.

The average time of nine $S$ s required for the 10 maps in each experiment is shown in Table 2. The time was longest in Exp. C, and more time was required in Exp. B than Exp. A. However, only the difference between Exp. A and $\mathrm{C}$ was significant at $5 \%$ level ( $t$-test).

It was noticed sometimes that the $S$ was conscious of a discrepancy between the direction that the $S$ thought correct and the direction that the guide lamp indicated. Hence, the $S$ was apt to be more perplexed in Exp. B (the dark room with a guide lamp) than Exp. C. In the latter, there was no cue to orientation except for the $S$ 's own feeling of direction.

(2) Comparisons among the maps: In Table 3 , the number of the $S$ s who made one or more errors in each map throughout all the experiments is given in the second column and the time required for a map, averaged over the $S$ s and experiments, is shown in the third column. The results indicated that the difficulty the $S$ met in following the path was not the same in all the ten maps. The largest number of errors were observed in the map No. 8 , and with respect to the time and errors, the $S$ seemed to have found the greatest difficulties in following maps No. 5, 8 and 9. Except for No. 6, the tendency was apparent that the time increased as the number of turns contained in the map increased. However, no such tendency was observed concerning the error. And the number of errors was not necessarily equal within the pair, No. 3 and No. 4, or No. 7 and No. 8, etc. though members of each pair have equal number of turns. The difficulty of the map seems to depend also upon other factors than the number of turns involved.

(3) Discussion: After the experiments $\mathrm{A}, \mathrm{B}$ and $\mathrm{C}$ were all finished, the $S$ was asked to describe introspectively the mental process going on during the performance. From these reports, it became apparent that the $S$ s could be divided into two groups with respect to the cue they used. Most of the $S \mathrm{~s}$ apprehended the map as a whole and tried to establish the correspondence between the map and the actual coordinates of the room before they started walking. The door and window of the room (in Exp. A), or the guide lamp (in Exp. B) served as the cue to the $S$ s of this type.

The $S$ s of the other type did not pay much attention to the relationship between the map as a whole and the room in advance, but determined the path to take whenever they came to the point to turn, taking into consideration only the direction and degree of the angle to turn. In short, their attention was limited to the part of the map pending decision on each occasion.

None the less, the difference between 
the two groups of the $S \mathrm{~s}$ in the mental process of orientation was not clearly reflected on the performances objectively scorable in terms of errors or time. Setting aside the question as to whether the $S$ apprehended the map partially or as a whole, it is still true that the $S$ had to effect a translation of the direction in the map into the corresponding directions in the room. That is to say, it was indispensable for the $S$ to rotate the map mentally in order to orient himself in Exp. I. For the purpose of analysing the process of this kind more precisely, Exp. II was conducted.

\section{EXPERIMENT II}

\section{Method}

(1) Subjects: Eight out of the nine $S$ s who participated in Exp. I served as subjects.

(2) Materials: Diagrams shown in Fig.2 were used which were similar, in the basic structure, to the maps in Exp. I. Each of the five diagrams was drawn on the white card-board ( $(10 \times 10 \mathrm{~cm}$. ) with black lines $0.5 \mathrm{~mm}$. in width.

(3) Procedures: One of the diagrams was presented in a frontal plane to the $S$, at the
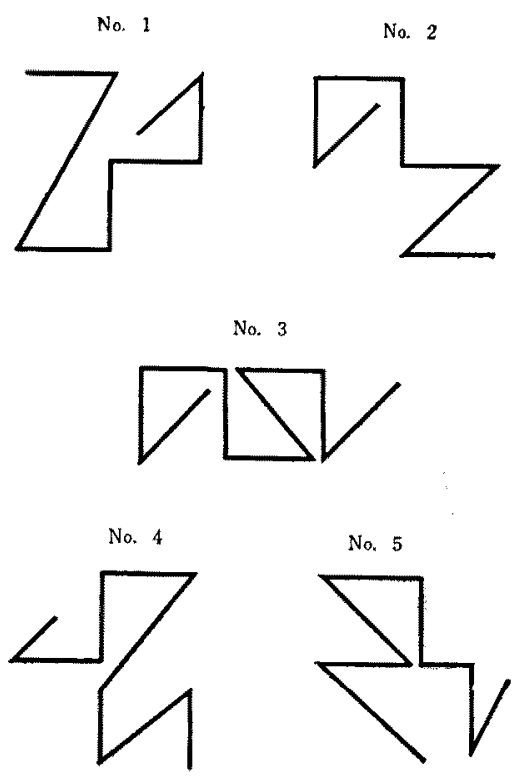

Fig. 2. The five diagrams used in Exp. II. distance of $40 \mathrm{~cm}$. from his eyes. The $S$ was asked, looking at the presented diagram, to describe it on the paper in rotated form in the manner instructed by the $E$. The rotations were (A) rotating $180^{\circ}$ in the frontal plane, (B) rotating $90^{\circ}$ to the right or the left in the frontal plane, $(\mathrm{C})$ rotating $180^{\circ}$ about the vertical axis, namely, describing the diagram as though it were observed from backside of the cardboard.

The $S$ was instructed to describe the rotated diagram as accurately and quickly as possible. Before the experiment was begun, the $E$ demonstrated the descriptions required in (A), (B) and (C) with a L-shaped diagram.

The experiment was conducted with each diagram in each of the three conditions of rotation. The diagrams were presented one by one in the order from diagram No. 1 to No. 5, but the order of the rotation (A), (B) and (C) was randomized. The time interval between the moment of presenting the diagram and the moment the $S$ began to describe the rotated form (the time required to construct the rotated diagram in mind), and the time from the beginning of drawing to its completion were recorded separately. Once the $S$ had finished the drawing, the correction of his errors was not permitted. The whole experiment was conducted in one sitting.

(4) Additional experiment: It must be examined first whether or not the time required for the $S$ to describe the diagram in the required form, apart from the time used to construct the rotated form in mind, is significantly different from diagram to diagram. For this purpose, the diagrams were presented one by one in such an orientation as required in the descriptions in (A) or (B) or (C) and the $S$ was instructed to describe the diagram as perceived (without rotating). The time required for it was recorded.

The $S \mathrm{~s}$ in this experiment were six university students, four males and two females. None of them participated in the preceding experiment.

\section{Results}

(1) Comparisons among the conditions: Table 4 shows the total number of errors made by all the $S$ s in each diagram under each rotation. No matter how many 
Table 4

The total number of errors in each diagram under each condition.

\begin{tabular}{c|c|c|cc}
\hline Diagram No. & Condition A & Condition B & Condition C \\
\hline 1 & 3 & 3 & 0 & 6 \\
2 & 1 & 2 & 0 & 3 \\
3 & 1 & 0 & 1 & 4 \\
4 & 1 & 2 & 0 & 2 \\
5 & 1 & 1 & 1 & 16
\end{tabular}

Table 5

The mean time, in sec. required to describe each diagram as perceived (additional experment) and that required to describe each diagram in rotated form.

\begin{tabular}{c|c|cc|c|c|c|c|c}
\hline \multirow{2}{*}{ Diagram No. } & \multicolumn{2}{|c|}{$\begin{array}{c}\text { mean time } \\
\text { of Cond. A }\end{array}$} & \multicolumn{2}{c|}{$\begin{array}{c}\text { mean time } \\
\text { of Cond. B }\end{array}$} & \multicolumn{2}{c}{$\begin{array}{c}\text { mean time } \\
\text { of Cond. C }\end{array}$} & \multicolumn{2}{c}{$\begin{array}{c}\text { mean time } \\
\text { of 3 Cond. }\end{array}$} \\
\cline { 2 - 8 } & add. exp. & Exp. II & add. exp. & Exp. II & add. exp. & Exp. II & add. exp. & Exp. II \\
\hline 1 & 6.4 & 51.0 & 6.4 & 27.9 & 5.9 & 22.7 & 6.1 & 33.9 \\
2 & 5.9 & 35.8 & 6.1 & 44.0 & 5.5 & 24.1 & 5.8 & 34.6 \\
3 & 7.4 & 56.2 & 7.1 & 35.9 & 7.7 & 26.1 & 7.4 & 39.4 \\
4 & 7.0 & 69.9 & 7.5 & 39.8 & 7.4 & 27.0 & 7.3 & 45.6 \\
5 & 6.8 & 46.0 & 6.6 & 58.8 & 7.3 & 25.3 & 6.9 & 43.4 \\
\hline mean time of & 6.7 & 51.8 & 6.7 & 41.3 & 6.8 & 25.0 & 6.7 & 39.4
\end{tabular}

errors occured in the description of a diagram, they were counted as one error. Thus, the maximum possible number of errors in each cell of Table 4 is 8 , i.e., the number of the $S_{\mathrm{s}}$.

As is apparent from Table 4, the number of errors was relatively small in general, and it is clear that the task to describe the rotated diagram was much easier under Condition (G) than under the others.

The mean time required for the $S$ to describe a diagram (from the presentation of a diagram to the finish of the drawing), in Exp. II as well as in the additional experiment, is shown in Table 5 .

Table 5 is divided into four sections each of which contains a pair of columns. The figures in each pair of columns represent the mean time required for drawing the same diagram under the two different conditions; the additional experiment and
Exp. II, in the latter the $S$ had to visualize the diagrams in rotated form prior to the start of drawing.

The time spent between the presentation of the diagram and the start of describing was approximately $30 \%$ of the time shown in the column "Exp. II".

From the results of the additional experiment, it became clear that, in so far as the mean time is concerned, there were no significant differences among the five diagrams and also among the three conditions.

In Exp. II, differences in the mean time were significant among the three conditions, decreasing in the following order: Conditions (A), (B) and (C). The difference between Conditions (A) and (C) was significant at $1 \%$ level ( $t$-test).

Taking into consideration the crror as well as the mean time, it seems to be conclusive that the rotation was easiest under 
Table 6

The median and quartiles of the time, in sec. required to describe a diagram in Exp. II.

\begin{tabular}{c|c|c|c}
\hline & Condition A & Condition B & Condition C \\
\hline median & 37.0 & 30.9 & 21.5 \\
$Q_{1}$ & 25.8 & 23.7 & 14.3 \\
$Q_{3}$ & 62.7 & 41.2 & 32.3 \\
\hline $\mathbf{Q}_{\mathbf{3}}-Q_{1}$ & 36.9 & 17.5 & 18.0
\end{tabular}

Condition (C). Of the time required to describe a diagrm in Exp. II, median and quartiles over the $S$ s are shown in Table 6. Since the interquartile range is largest in Condition (A), the individual difference seems to appear most distinctly when the $S$ described the diagram rotated by 180 degrees.

(2) Comparisons among the diagrams: Errors were made more frequently in the diagrams No. 1 and No. 4 than in the others (Table 4). Though variation in the mean time in Exp. II was not significant statistically among the diagrams, the time was largest in diagram No. 4 throughout all the conditions. It is worthy to note that diagram No. 4 corresponds in its structure to No. 8 of Exp. I and it was in this map that the greatest number of errors was observed in Exp. I (Tables 3, 5).

(3) Discussion: It would be impossible to point out with confidence from these results alone factors responsible for making the mental rotation of the diagrams difficult. It is supposed, however, that there are some common factors equally responsible for the difficulty of the task in Exp. I and that in Exp. II. Hence, it might be well to make a brief mention of the relationship between the two. Some consistent individual differences were observed among the eight $S_{\mathrm{s}}$ who participated in both Exps. I and II. Rank correlation of the number of errors over the $S$ s between Exp. I and II was fairly high $(\rho=0.84)$. Whereas rank correlation of time was rather low $(y=0.38)$. Hence, it might be concluded that, with respect to difficulty expressed in terms of errors, a consistent tendency existed between the process of orienting oneself in the room and the process of orientation in reproducing visually presented diagrams in rotated form.

\section{SUMmary}

The purpose of the present study was to investigate two kinds of orientation: The one was to orient oneself correctly while walking and the other was to reproduce visually presented diagrams in rotated form.

In Exp. I, the $S$ was asked to walk in the room along the path indicated in the map which the $S$ was holding, while walking, in a constant relation to the $S$ 's body. As the $S$ made turns, it happened quite often that the map was not in the proper orientation to the room. The $S$ performed the task in three cxperiments : (A) in a normally illuminated room, (B) in a dark room with a guide lamp on the wall, $(\mathrm{C})$ in a completely dark room. The task was most difficult in the Exp. $\mathrm{C}$ and easiest in the Exp. A when it was expressed in terms of the error made and the time required. A guide lamp in Exp. B was not so useful as a cue for the orientation, though it proved of some use in controlling the angle to turn.

In Exp. II, the $S$ was instructed to observe a diagram visually presented and describe its rotated form in the manner indicated by the $E$. The rotations were as follows: (A) rotating $180^{\circ}$ to the right or the left in the frontal plane, (B) rotating $90^{\circ}$ to the right or the left in the frontal plane, (C) rotating $180^{\circ}$ about the vertical axis, namely, describing the diagram as though it were observed from the backside of the cardboard. According to the error and time, the difficulty of the task was in the following order : Conditions (A), and (B) and (C). And the task seemed to be particularly easy in Condition $(\mathrm{C})$.

From the results of Exp. I and II, it was suggested that there were some common factors responsible for the difficulty of ori- 
entation in Exp. I as well as in Exp. II, which was shown by the rank correlation $(\rho=0.84)$ of the error between the two experiments over the $S$ s who participated in both.

\section{REFERENCES}

Angyal, A. Uber die Raumlage vorgestellter Örter. Arch. ges. Psychol., 1930, 78, 47-94.

Claparède, E. La faculté d'orientation, sens de direction, sens de retour. Arch. Psychol., Geneve, 1903, 2, 133-180.

Claparède, E. Note sur la localisation du moi. Arch. Psychol., Genève, 1924, 19, 172-182.

ClAPARÈDE, E. L'orientation lointain. Nouveau Traite de Psychologie. VIII, 3. Paris: 1943.
Fisher, M. H. Die Orientierung im Raume bei Wirbeltieren und beim Menschen. Handbuch der norm. und path. Physiol. XV/2. Berlin : 1931.

Ryan T. A., \& Ryan M. S. Geographical orienta-i] tion. Amer. 7. Psychol., 1940, 53, 204-215.

SAndström, C. I. Orientation in the present space. Stockholm: Almqvist \& Wiksells, 1951.

Semmes, J., Weinsten, S., Ghent, L., \& Teuber, H. Spatial orientation in man after cerebral injury. I. Analyses by locus of lesion. $j$. Psychol., 1955, 39, 227-244.

$\mathrm{S} f \mathrm{~A}$ o, $\mathrm{C}$. The further study of orientation in the perception of space. Jap. J. Psychol., 1960, 31, 153-160, (in Japanese).

(Received April 13, 1960) 